\title{
HIZB UT TAHRIR EN ASIA CENTRAL A COMIENZOS DEL SIGLO
} XXI

\author{
Antonio Alonso ${ }^{1}$ \\ UNISCI/Universidad CEU San Pablo
}

\begin{abstract}
Resumen:
El movimiento islamista Hizb ut Tahrir se ha extendido por todo el mundo desde que fue fundado por Taquiuddin al Nabhani en los años 50. Su presencia en Asia Central ha contribuido a desestabilizar a las naciones que hace apenas 20 años se independizaron y a incrementar el número de islamistas que, posiblemente, pueden dar el salto a grupos de otra índole, esto es, terroristas. El grupo se ha fortalecido en los últimos años en todo el mundo y durante 2011 ha estado muy presente en las revueltas de la primavera árabe.
\end{abstract}

Palabras clave: Hizb ut Tahrir, Asia Central, Taquiuddin al Nabhani, primavera árabe.

Title in English: "Hizb Ut Tahrir in Central Asia at the Beginning of the XXIst Century".

\begin{abstract}
:
Islamist movement Hizb ut Tahrir has expanded his activities worldwide since it was founded by Taquiuddin al Nabhani in the 50's. Its presence in Central Asia has contributed to destabilize those nations which became independent 20 years ago and to increase the number of Islamists who, plausibly, can jump to groups of different nature, i.e. terrorist. The group has been reinforced in recent years all over the world and during 2011 was very active in the Arab Spring's revolts.
\end{abstract}

Keywords: Hizb ut Tahrir, Central Asia, Taquiuddin al Nabhani, Arab Spring.

Copyright (C) UNISCI, 2012.

Las opiniones expresadas en estos artículos son propias de sus autores, y no reflejan necesariamente la opinión de UNISCI. The views expressed in these articles are those of the authors, and do not necessarily reflect the views of UNISCI.

\footnotetext{
${ }^{1}$ Antonio Alonso Marcos es profesor del Instituto de Humanidades "Ángel Ayala”, Universidad CEU-San Pablo, e investigador de UNISCI. Dirección: Instituto de Humanidades "Ángel Ayala", Universidad CEUSan Pablo, Paseo Juan XXIII, 8, 28040, Madrid.

Madrid, España. E-mail: aalonso@ceu.es.

http://dx.doi.org/10.5209/rev_UNIS.2012.n28.3840u
} 


\section{Introducción}

Cuando en 1991 la Unión Soviética desapareció, las cinco repúblicas de Asia Central (Kazajstán, Kirguistán, Tayikistán, Turkmenistán y Uzbekistán) tuvieron que manejar todos los asuntos que antes se llevaban desde Moscú, tanto en política interior como exterior. Uno de los principales problemas que afectan a esta inmensa región que hace de colchón entre Rusia y la inestable zona de Irán, Afganistán y Pakistán, es la del islamismo radical que rápidamente se transforma en violencia terrorista.

Hizb ut Tahrir al Islami (Partido de la Liberación Islámica) es el movimiento islamista más extendido por distintos países, que cuenta con gran cantidad de seguidores de diferente nivel de compromiso.

\section{20 años de independencia}

Un vistazo a la región nos ofrece un panorama poco homogéneo en cualquiera de los aspectos (político, económico, social, religioso,...). Las diferencias son tan grandes de país a país que apenas se podría imaginar que las cinco repúblicas eran parte hace apenas 20 años del mismo Estado. Aunque culturalmente se pueden observar ciertas similitudes (la forma de honrar a los invitados, su hospitalidad, su mentalidad tradicionalista,...) los indicadores ofrecido por cualquier institución (el Banco Mundial Freedom House o el PNUD, entre otros) nos muestran cinco realidades bien diferentes.

En términos generales, se podría decir que el país más avanzado y donde se disfrutan más libertades es Kazajstán. Uzbekistán le seguiría en desarrollo económico pero no en el plano político, aventajado por Kirguistán (aunque este es un país que ha sufrido fuertes turbulencias en los últimos diez años, con dos revoluciones de por medio). Tayikistán es el país más pobre y Turkmenistán, a pesar de contar con inmensas bolsas de gas, no acaba de salir del autoritarismo político al que le somete Berdimujamedov.

A grandes rasgos podemos decir que la campaña liderada por EE.UU. en Afganistán supuso un antes y un después para la región y que, en general, la presencia norteamericana ha sido un factor determinante para la configuración del subsistema regional ya que ha sido uno de los elementos más importantes en torno al cual han debido acomodarse otros asuntos de gran interés ${ }^{2}$.

La actual crisis económica y financiera también ha sacudido a esta región aunque, como es evidente, de manera muy distinta a cada país. Aparte de las grandes disparidades en cuanto al PIB, el desarrollo y liberalización de las economías también es muy distinto en Kazajstán, donde está muy avanzado, o Uzbekistán, que ha iniciado una serie de reformas que le permiten captar más inversión extranjera, que la situación en Kirguistán, que depende prácticamente del negocio de la importación-exportación de productos chinos, o Turkmenistán, que depende del sector energético. Tayikistán es quien se lleva la peor parte ya que, además de partir con la desventaja de una guerra civil (1992-97), la situación no ha prosperado mucho y es, con diferencia, el país más empobrecido y con mayor tasa de paro (especialmente paro juvenil).

\footnotetext{
${ }^{2}$ Sobre la situación general de Asia Central y los atores involucrados en este espacio geopolítico ver Stavridis, Stelios y De Prado, César (coords.) (2010): Panorámica de actores y factores en Asia Central, Zaragoza, Universidad de Zaragoza. Ver también la obra más reciente de Veiga, Francisco y Mourenza, Andrés (coords.) (2012): El retorno de Eurasia, 1991-2011: Veinte años del nuevo gran espacio geoestratégico que abrió paso al siglo XXI, Barcelona, Península.
} 
La situación política es también muy distinta dependiendo del país en el que nos fijemos. Así, podemos ver que existen regímenes que aspiran a ser plenamente democráticos ${ }^{3}$ y que realizan un trabajo progresivo, aunque muy lento, hacia una democracia más participativa, con mayor protagonismo de la sociedad civil, como es el caso de Kazajstán ${ }^{4}$. Uzbekistán ha sido denunciado en numerosas ocasiones por casos de tortura y de violación de los Derechos Humanos; sin embargo, cuenta con elecciones libres en las que compiten diversos partidos y varios de ellos obtienen amplia representación en la Cámara Baja ${ }^{5}$. Ambos países son dirigidos por los mismos presidentes desde el inicio de la independencia, con cierto éxito hay que decir, lo que a la vez plantea una serie de problemas a la hora de preguntarse acerca de la sucesión que no se prevé nada fácil ${ }^{6}$.

El mismo problema se plantea en Tayikistán, donde a pesar de los problemas económicos y de las escasas libertades la situación permanece estable, sólo asediados por las amenazas provenientes de Afganistán (principalmente droga e islamismo radical y paso de terroristas).

En Turkmenistán fue la naturaleza quien resolvió ese problema ya que el anterior dictador, Niyazov, murió repentinamente en diciembre de 2006 y fue sucedido sin grandes problema por su supuesto hijo, Berdimujamedov.

Kirgusitán es el país que más problemas ha tenido en este ámbito, pues ha sufrido dos revoluciones populares, una en 2005 y otra en 2010, que acabó con el derrocamiento de sendos presidentes, Akayev y Bakiyev. La instauración en 2010 de un gobierno provisional, con un Jefe de Estado también provisional, Reza Otombayeva, también fue traumática, ya que se produjeron una serie de disturbios en el sur del país, al parecer instigados por familiares directos del expulsado Bakiyev, que causaron grandes destrucciones materiales, decenas de muertos y miles de refugiados ${ }^{7}$. Las reformas constitucionales y la elección de un nuevo presidente en diciembre de 2011, Almazbek Atambayev, parece que han servido para traer estabilidad a este pequeño pero convulso país ${ }^{8}$.

\footnotetext{
3 "Notwithstanding the government's stated ambition to strengthen Kazakhstan's democratic processes and to conduct elections in line with international standards, the 15 January early parliamentary elections still did not meet fundamental principles of democratic elections. They were technically well administered and certain legal changes were passed, aimed at introducing at least a second party into the parliament; yet, the necessary conditions for the conduct of genuinely pluralistic elections, which are a prerequisite for functioning democratic institutions, were not provided for by the authorities". Ver el Informe de la OSCE: "Statement Of Preliminary Findings And Conclusions", OSCE/ODIHR Election Observation Mission, 16 de enero de 2012, en http://www.osce.org.

4 Ver Alonso Marcos, Antonio: "Observación electoral OSCE desde Taraz (Kazajstán)", Boletín del Observatorio Asia Central, $\mathrm{n}^{\circ} 19$ (enero de 2012), en http://www.asiacentral.es.

5 "The OSCE/ODIHR has never deployed a full fledged election observation mission to Uzbekistan due to a lack of minimum conditions for democratic elections. Previous elections were assessed as generally falling short of OSCE commitments. The 27 December 2009 parliamentary elections took place in the context of Uzbekistan's declared "step-by-step approach" towards further democratization. While some of the December 2008 amendments to the election law slightly improve the legal framework for elections, their good faith implementation remains indispensable to ameliorate electoral practices in Uzbekistan". Ver el informe de la OSCE: "Republic Of Uzbekistan. Parliamentary Elections, 27 December 2009 OSCE/ODIHR Election Assessment Mission Final Report”, OSCE/ODIHR, 7 de abril de 2010, en http://www.osce.org, p. 1.

${ }^{6}$ Ver, entre otros, el "Documento de análisis: Visión Geopolítica de los resultados de las elecciones 2012. Enero.”, Instituto Español de Estudios Estratégicos, Documento de Análisis 07/2012 (1 de febrero de 2012), en http://www.ieee.es.

${ }^{7}$ Ver de Pedro, Nicolás: “Kirguizistán: crisis y expectativa democrática”, Real Instituto Elcano, ARI 118/2010 (14 de julio de 2010), en http://www.realinstitutoelcano.org.

8 Ver Ruiz Ramas, Rubén: "Kirguistán/ Por qué estas elecciones son relevantes y por qué no lo son", Observatorio Político y Electoral del Mundo Árabe y Musulmán, 20 de octubre de 2011, en http://www.opemam.org. Ver también Ruiz Ramas, Rubén: “Kirguistán / Elecciones Presidenciales, 30 de
} 
En el capítulo de la convivencia social, el modelo kazajo debe ser resaltado. Al igual que sus vecinos, cuenta en su interior con un gran número de minorías étnicas (más de 130) y confesiones religiosas (más de 40). Pero a diferencia de lo que ocurre en los demás Estados, en Kazajstán se protege desde la propia Constitución el modelo de tolerancia y de convivencia, de manera que ningún grupo puede reclamar la superioridad étnica ni emprender acción alguna dirigida a ofender a las demás, menos aún si se emplean los medios de comunicación 9 .

Esta zona del mundo ha sido codiciada desde tiempos remotos por los grandes imperios y por las grandes potencias del momento. La actualidad no es una excepción a esta norma básica en Asia Central y se puede constatar una lucha soterrada por hacerse con la influencia política sobre estos países y por el control de sus recursos naturales, especialmente los energéticos. En esta carrera participan, en primer lugar, Rusia, EE.UU. y China. Compiten en un segundo nivel otras potencias medias como son Irán, India, Turquía, Japón, Corea del Sur o la UE. De entre los países de la UE Alemania, Francia, Reino Unido e Italia son quienes están más presentes en la zona; España queda relegada a un modestísimo papel, aunque ha hecho significativos avances recientemente, sobre todo en el ámbito ferroviario ${ }^{10}$.

Las repúblicas centroasiáticas han tenido que atender a estos pretendientes de manera más o menos ordenada. Sin embargo, los acontecimientos de 2001 supusieron un inciso en esa armonización orquestada y se vieron "obligados" a prestar ayuda a EE.UU. Sin embargo, el año 2005 supuso una vuelta hacia Rusia y China, orientación que ha permanecido al menso hasta el final de la Administración Bush y bien entrada la de Obama.

Por otro lado, la región busca ayuda en los "grandes" en materia de seguridad, lo que se ve reflejado en las distintas alianzas o acuerdos de carácter militar que se han firmado en los últimos 20 años: CEI, OTSC, GUUAM, OCS e incluso la Asociación para la paz de la OTAN.

Las amenazas a las que tiene que hacer frente son de diferente índole. Si entendemos el término en sentido estricto, es decir la voluntad manifiesta de hacer daño a un ente, vemos que persiste el radicalismo, el extremismo y el terrorismo. Si tomamos el sentido amplio del término, podremos incluir los distintos tráficos ilegales transnacionales (droga, armas, personas), los riesgos medioambientales (inundaciones, sequías, desecación del mar de Aral), la falta de abastecimiento energético (que afecta principalmente a Kirguistán y Tayikistán). La situación económica y la falta de oportunidades y de formación para los más jóvenes también pueden ser consideradas un peligro para la estabilidad de la región. La corrupción política y las corruptelas de los cuerpos de seguridad también son asuntos de máxima preocupación pues están conectados a otras cuestiones que causan inseguridad en la zona (como el narcotráfico) o inestabilidad (la falta de avance en una democracia real).

Aunque puede parecer que alguno de estos factores es problema de un solo país, en realidad afecta a toda la región, pues una situación deteriorada en uno de estos Estados afecta negativamente al resto, generando inestabilidad regional. Por ejemplo, aunque los problemas económicos afecten sólo a Tayikistán de manera devastadora, esto afecta a Kazajstán, pues, como nación más rica de la zona, recibirá mayor afluencia de inmigrantes. La inestabilidad

octubre de 2011", Observatorio Político y Electoral del Mundo Árabe y Musulmán, 16 de diciembre de 2011, en http://www.observatorioelectoral.es.

${ }^{9}$ Ver Alonso Marcos, Antonio (2011): Kazajistán: Modelo de tolerancia religiosa, Madrid, UNISCI.

${ }^{10}$ Ver Bonet, Pilar: "Uzbekistán ningunea a España en la puesta en marcha del AVE", El País, 30 de agosto de 2011, en http://www.elpais.com.

Ver "Talgo abre fábrica en Astaná", Observatorio de Asia Central, 7 de diciembre de 2011, en http://www.asiacentral.es. 
política de Kirguistán provocó una avalancha de refugiados en Uzbekistán. Y así sucesivamente.

\section{Hizb ut Tahrir al Islami (Partido de la Liberación Islámica)}

\subsection{Taquiuddin al Nabhani}

El Sheikh Muhammad Taqiuddin bin Ibrahim, bin Mustafah, bin Ismail, bin Yusuf alNabhani, fundó el movimiento islamista Hizb ut Tahrir al Quds entre 1952 y 1953 junto con otros compañeros, entre ellos Dawud Hamdan, Ghanim Abduh, Munir Shuqayr y Adil alNablusi. Su familia pertenecía a una prestigiosa saga de intelectuales expertos en Derecho (en la ley islámica, se entiende). Se crió, por tanto, rodeado de un ambiente que le impulsó a profundizar en los conocimientos de la parte jurídica del Islam, que es uno de los ejes principales de dicha religión ${ }^{11}$. Amplió sus estudios en los años 20 y 30 en la Universidad de Al- Azhar (El Cairo), participando de un ambiente muy convulso en le que surgieron, entre otros, los Hermanos Musulmanes e ideólogos como Sayid Qutb o Hasan Al Bana.

A su vuelta a la Palestina bajo mandato británico se integra en los tribunales de justicia (islámica) de Jerusalén, pero la salida del Imperio Británico de la zona y la lucha por el poder y el posterior establecimiento del Estado de Israel hacen que se implique cada vez más en política, de manera que algunos biógrafos sitúan le sitúan en los años 40 en la lucha contra el poder establecido en Siria, llegando incluso a conspirar para llevar a cabo golpes de Estado con el Partido Ba'th (Hizb ul-Ba'th) ${ }^{12}$.

Esa experiencia le impulsa a crear en los años 50 el partido y a poner por escrito gran parte de su pensamiento ${ }^{13}$. A finales de 1952, siguiendo la Ley de Sociedades vigente (del Imperio Otomano), enviaron una nota al gobernador de Jerusalén y al gobierno jordano, informando a las autoridades de la fundación del partido. Sin embargo, el gobierno jordano reaccionó al instante y publicó un decreto por el que se prohibió el partido, declarando ilegales todas sus actividades. No obstante, al Nabhani ignoró dicha orden y continúo sus actividades en Jerusalén, al-Khalil, Nablus y otras ciudades.

En los años 60 participó en muchas de las luchas por la independencia real de las nuevas naciones descolonizadas y se dedicó a afianzar el partido y su estrategia para implantar el califato a través de tres etapas: una de estudio, de cambio cultural; una segunda de interacción con la sociedad; y una tercera de toma del poder.

Al Nabhani murió en Beirut el 20 de diciembre de 1977 y fue enterrado en el cementerio de al-Auza'i ${ }^{14}$. Le sucedió Abdul Qadeem Zallum, quien había sido compañero

\footnotetext{
${ }^{11}$ Ver Khalil Samir, Samir (2003): Cien preguntas sobre el Islam, Madrid, Encuentro.

${ }^{12}$ Abdullaah at-Tall fue un árabe nacionalista del partido Ba'th. Éste envió a Damasco, como parte de un plan para un golpe militar, a as-Sayyid Abdullaah ar-Rimaawee y a an-Nabahani. Les dio su pasaporte político para que pudieran presentarlo al líder, Husni az-Za'eem, ya que este era el gesto que habían convenido entre ellos. Así, ar-Rimaawee y an-Nabhani pasaron a Damasco el 7 de mayo de 1949 con la excusa de comprar papel para la publicación de la Revista "Ba'th". Ver Al-Musali, Ahmad (2004): Encyclopedia of Islamic Movements in the Arabic Nation, Turkey and Iran, Beirut, Center for the Studies of Arab Unity, p. 407.

13 A esta época pertenecen: Salvar a Palestina (1950); El Mensaje de los árabes (1950); El Sistema para la sociedad (1950); El sistema del Islam (1953); El sistema de gobierno en el Islam (1953); El sistema económico en el Islam (1953); El sistema social en el Islam (1953); La estructura del partido (1953); Los conceptos de Hizb ut-Tahrir (1953); y El Estado islámico (1953).

${ }^{14}$ Ver "Sheikh Muhammad Taqiuddin al-Nabhani “, 23 de agosto de 2009, en http://hizb-america.org. Otros autores sitúan la fecha de su muerte en 1979, lo cual es poco probable. Ver la entrada "al-Nabhani, Taqi al Din. Islamic militant” en Mattar, Philip (2005): Encyclopedia of the Palestinians, Nueva York, Infobase Publishing, p. 326.
} 
del fundador. Éste se mantuvo en su puesto hasta que dimitió por problemas de salud en 2003, muriendo apenas dos meses después. Zallum dejó algunos documentos importantes tales como La campaña Americana para suprimir el Islam; La democracia es un sistema infiel; La cruzada de George Bush contra los musulmanes; La metodología de Hizb ut-Tahrir para el cambio; y La inevitabilidad del choque de civilizaciones.

En 2003 Ata Abu Rashta, también antiguo compañero de al Nabhani, tomó las riendas del partido, un partido muy dividido, con muchas escisiones ${ }^{15}$, pero que aún mantenía ese liderazgo global.

La importancia del fundador se puede observar aún en los círculos de estudio y en los comunicados de la organización pues son sus obras lo que se estudia y se discute en ellos, no el Corán ni ningún otro libro sagrado. Se subraya así la naturaleza no religiosa sino política (en sentido amplio de la palabra, es decir, "social") de este movimiento de barniz islámico ${ }^{16}$.

\subsection{Su ideología}

El objetivo de este movimiento islamista de base suní es crear un califato ${ }^{17}$, una especie de estado transnacional, en cuyo seno se reunirían distintas naciones, puestas todas bajo la dirección de un califa elegido por el pueblo. Este califa gobernaría basándose en la sharía y emprendería una yihad contra el resto de naciones que estuvieran fuera de este califato para someterlas políticamente; tal sería su principal instrumento en Política Exterior ${ }^{18}$.

Aunque ya se ha escrito mucho sobre este tema, la ideología de Hizb ut Tahrir es sibilinamente violenta ya que llena de odio y rencor la mente de los musulmanes que le prestan atención, buscando impactar especialmente en la mente de los jóvenes ${ }^{19}$. Además, es importante subrayar que rechaza de plano la democracia por ser un sistema infiel, distinto al que, según su lectura, es el exclusivo del Islam: el califato. Así, la democracia sería un producto occidental, residuo del colonialismo, herramienta de este para perpetuar su presencia en territorio musulmán, y no podría aportar nada bueno al buen gobierno de los musulmanes.

\subsection{Su modus operandi}

$\mathrm{Su}$ forma de actuar es distinta dependiendo, principalmente, de dos factores: 1) de si se trata de un país mayoritariamente musulmán; y 2) de si disfruta de un marco legal que proteja las libertades y los derechos fundamentales. No obstante, la estructura del partido no varía mucho de un lugar a otro, simplemente depende del número de simpatizantes y militantes con los que cuente.

La célula básica de la organización es el círculo de estudio. Formado por entre 5 y 7 personas, sólo el formador o responsable conoce al líder de esa ciudad (o barrio, dependiendo

\footnotetext{
${ }^{15}$ Ver Alonso Marcos, Antonio: “Análisis del discurso de Hizb ut Tahrir”, Athena Intelligence Journal, vol. 3, nº 2 (2008), pp. 99-101.

${ }_{16}$ Ver los distintos videos colgados bajo el título "Hizb ut-Tahrir Central London Circle" en http://www.youtube.com.

${ }^{17}$ Ver Ahmed, Houriya y Stuart, Hannah (2009): Hizb ut-Tahrir: Ideology and Strategy, Londres, The Centre for Social Cohesion.

${ }^{18}$ Ver el "Proyecto de Constitución" que se encuentra dentro del libro de al Nabhani, Taquiuddin (1998): El Estado Islámico, Londres, Al Khilafah Publuications, p. 267. Ver también el aparte dictado a la Política Exterior (arts. 176-186) en las pp. 273-276.

${ }^{19}$ Las campañas en las universidades británicas han sido objeto de estudio en varias ocasiones. Ver Simcox, Robin; Stuart, Hannah y Ahmed, Houriya (2010): Islamist Terrorism: The British Connections, Londres, The Centre for Social Cohesion. Ver también Thorne, John y Stuart, Hannah (2008): Islam on Campus: A survey of UK student opinions, Londres, The Centre for Social Cohesion. Por ultimo, ver Murria, Douglas (2010): Radical Islam on UK Campuses: A Comprehensive List of Extremist Speakers at UK Universities, Londres, The Centre for Social Cohesion.
} 
del volumen de militantes). Durante unos dos años, cada neófito debe empaparse de la doctrina de al Nabhani y demostrar que está concienciado de la necesidad del establecimiento del califato y demostrar su compromiso con la causa (asistiendo regularmente a los círculos de estudio, repartiendo panfletos, asistiendo a manifestaciones, procurando apoyo logístico y/o financiero a la organización,...). Los líderes de barrio o de ciudades se reúnen entre sí para coordinar sus actividades en aquella región y para recibir instrucciones del líder nacional ("provincial" en terminología de Hizb ut Tahrir, pues el califato, y la organización global, está dividido en "provincias", que se corresponden con los actuales Estados). Los líderes "provinciales" reciben las instrucciones, a su vez, del comité asesor del líder global.

Como se puede observar, se trata de una organización muy jerarquizada, bien planificada, en la que no caben acciones individuales libres, autónomas, sin permiso del líder global. Así, es difícil imaginar cómo una persona que ha militado en Hizb ut Tahrir no haya siquiera comunicado a sus superiores que abandona la organización definitivamente ${ }^{20}$ o para integrarse en un grupo terrorista.

Parece bastante evidente, pues, que Hizb ut Tahrir, aun no cometiendo directamente atentados terroristas, es parte de una cadena de montaje, de una división del trabajo del mundo islamista, en el que cada grupo tiene una función bien determinada (unos reclutan a los militantes o combatientes, otros les proporcionan pasaporte, otros recaudan fondos, otros se encargan del entrenamiento, otros dan cobertura logística, otros realizan campañas mediáticas, otros aportan pisos francos, unos se hacen con los explosivos, ... y otros ponen las bombas).

Aunque es cierto que Hizb ut Tahrir afirma rechazar el cambio de régimen a través de la violencia, se ha encontrado abundante material en casa de algunos de sus miembros al ser detenidos. Entre los objetos hallados había libros, panfletos, CD, DVD, ... pero también armas y explosivos $^{21}$. No obstante, la información sobre estos utensilios es poco fiable pues procede de la policía de países no plenamente democráticos $\mathrm{y}$, por lo tanto, debe ser tomada con sumo cuidado, ya que se puede tratar de "pruebas plantadas" por las propias fuerzas del orden y no de pruebas auténticas.

Su objetivo en los países occidentales no es el cambio de régimen, sino la búsqueda de musulmanes que puedan apoyar su causa de alguna manera, sea entre las élites del país (que sería lo preferible para ellos, aunque poco probable) o sea entre la base social (principalmente inmigrantes o descendientes de inmigrantes en busca de una identidad cultural definida). En estos países suelen operar abiertamente, manifestando a las claras cuales son sus objetivos y sus opiniones, dado el galantismo que existe en dichos sistemas jurídicos ${ }^{22}$. Gracias a esto, se puede recabar de manera fácil y accesible información fiable sobre la doctrina o la opinión del grupo. Además de las conferencias, lecciones, libros y panfletos, en cada país hay una página $w e b$ en lengua vernácula que posibilita el acceso rápido al mensaje de esta organización ${ }^{23}$.

\footnotetext{
${ }^{20}$ Ver Husain, Ed (2007): The Islamist: Why I Joined Radical Islam in Britain, what I Saw Inside and why I Left, Londres, Penguin.

${ }^{21}$ Ver Kamalov, Erkin: “Kyrgyz seize arms, extremist literature”, Central Asia Online, 14 de diciembre de 2011, en http://centralasiaonline.com.

22 "El Tribunal Europeo de Derechos Humanos dictaba sentencia el 17 de enero a favor del británico de origen jordano Omar Othman (alias Abu Qutada), en contra del Reino Unido". Ver GEES: "Garantismo europeo e islamismo radical", Libertad Digital, 26 de enero de 2012, en http://www.libertaddigital.com.

${ }^{23}$ La web oficial del movimiento en Australia (Hizb ut-Tahrir Australia) recoge los diversos links de las webs oficiales: Hizb ut-Tahrir, Hizb ut-Tahrir Media Office, Hizb ut-Tahrir Britain, Hizb ut-Tahrir Pakistan, Hizb utTahrir Bangladesh, Hizb ut-Tahrir Indonesia, Hizb ut-Tahrir Malaysia, Hizb ut-Tahrir Denmark, Hizb ut-Tahrir America, Hizb ut-Tahrir Morocco, Hizb ut-Tahrir Spain, Hizb ut-Tahrir Ukraine, Hizb ut-Tahrir Turkey, Hizb

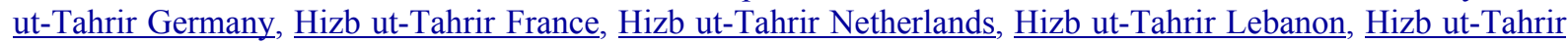
$\underline{\text { Palestine, Hizb ut-Tahrir Afghanistan, }}$
} 
En los países de mayoría musulmana, sin embargo, su forma de actuar es distinta, ya que tienen un numeroso auditorio potencial y hay más probabilidades de encontrar entre las élites personas que comulguen con su objetivo (establecer el califato) y con su metodología (de las tres etapas). En estas regiones deben andarse con más cuidado pues suelen tratarse de regímenes poco tolerantes, bastante férreos, donde los derechos y libertades fundamentales apenas están protegidos.

\section{4. Su presencia en el mundo}

\subsubsection{Cuartel general}

Como es bien sabido, la oficina central del movimiento global se encuentra en Londres ${ }^{24}$. Allí es donde desarrolla su actividad más visible y desde donde organiza grandes manifestaciones públicas, especialmente cada vez que un dirigente visita el Reino Unido. También coordina desde allí las actividades del movimiento en todo el mundo. Sin embargo, se cree que el líder del movimiento, Ata Abu Rashta, reside en el Líbano ${ }^{25}$.

Los miembros del Comité Ejecutivo desarrollan una activísima labor en los medios de comunicación para hacer presente a su organización en medio de la sociedad británica (aunque también lo hacen en Australia). Estos miembros son: Abdul Wahid (Presidente Ejecutivo del Partido en el Reino Unido), Taji Mustafa (Representante ante los medios de comunicación), Imran Waheed (Asesor Jefe para las relaciones con los medios), Nasreen Nawaz (Representante de las mujeres del partido ante los medios), Jamal Harwood (Jefe de los asuntos legales) y Sajjad Khan (Asesor Jefe de asuntos políticos) ${ }^{26}$.

Londres sufrió una serie de atentados terroristas en dos días distintos de julio de 2005 (el 7 y el 21). Algunos de los terroristas tenían vínculos con el movimiento Hizb ut Tahrir, lo que hizo que el Primer Ministro, Tony Blair, intentara ilegalizarles. Finalmente, tras un largo proceso y de debates parlamentarios, tanto en la Cámara de los Comunes como en la de los Lores, se desaconsejó la ilegalización de la organización y se mantuvo su status. De nuevo en 2007, una serie de atentados sacudieron la sociedad británica con el atentado al aeropuerto de Glasgow, donde un terrorista suicida fue capturado vivo y se pudo comprobar su vinculación, de nuevo, con Hizb ut Tahrir. A pesar de los informes, provenientes de organismos públicos y privados, que aconsejan que este grupo sea perseguido, parece que seguirá operando libremente por mucho tiempo en el Reino Unido.

\subsubsection{Países no islámicos}

Este movimiento ha sido prohibido en algunos países, como Alemania, Dinamarca y Rusia por promover el odio antisemita y contra el Estado de Israel, a quien considera un enemigo con el que está en guerra ${ }^{27}$. En Holanda fue puesto bajo sospecha por los servicios de

\footnotetext{
${ }^{24}$ Más concretamente en: "Hizb ut-Tahrir Britain, Suite 301, 28 Old Brompton Road, London, SW7 3SS, UK Telephone: 07074-192400,E-mail press@hizb.org.uk”. Asi aparece en el Hizb ut-Tahrir Britain: "Media Information Pack", en http://www.hizb.org.uk.

${ }^{25}$ Ver Alonso Marcos, Antonio: "Hizb ut Tahrir en el Líbano: Sus aportaciones al islam político", Revista CIDOB d'Afers Internacionals, no 93-94, p. 202.

${ }^{26}$ Ver el "Media Information Pack", op. cit., p. 11.

27 "[...] countries which are in a condition of war with us, such as Israel". Ver el art. 157 del "Proyecto de Constitución”, op. cit., p. 270. Y, sobre todo: “Art. 184. The State's relationships with other states are built upon four considerations. These are: [...] 4. With states that are actually belligerent states, such as Israel, a state of war must be taken as the basis for all dispositions with them. They must be dealt with as if a real war existed between us, whether during cease fire or other wise. All citizen of such states are prevented from entering the State". Ibidem, p. 275.
} 
inteligencia $^{28}$, de manera que periódicamente realiza informes sobre ellos, afirmando en 2011 que

In the Netherlands, this organisation has always remained within the bounds of the law and democratically acceptable activity. It attempts to attract well-educated Muslims by using a modern style of Islamic reasoning. Last year, for example, it argued that participating in elections - be it active or passively - is un-Islamic. From the response this generated in the media and on the Internet, it is clear that that idea is firmly rejected by many individual Dutch Muslims and by umbrella organisations like the Union of Moroccan Mosques in the Netherlands (Unie van Marokkaanse Moskeeorganisaties in Nederland, UMMON) and the Contact Body of Muslims and the Government (Contactorgaan Moslims en Overheid, CMO). This demonstrates that there is considerable resistance to the Hizb ut-Tahrir message within the community. ${ }^{29}$

En Dinamarca han abogado abiertamente por boicotear entre los ciudadanos musulmanes las elecciones democráticas, en su línea de ir contra este sistema impuro ${ }^{30}$.

En España parece que no tiene mucha fuerza, aunque busca adeptos en los ambientes antisistema de Cataluña, especialmente entre la población pakistaní, y en el sur de España entre los marroquíes ${ }^{31}$.

También tienen cierta presencia en Polonia y Ucrania, y son muy activos en Asutralia y EE.UU., donde han celebrado varias conferencias de numeroso auditorio ${ }^{32}$.

\subsubsection{Países islámicos}

Como se ha dicho anteriormente, Hizb ut Tahrir actúa de distinta manera en lso países musulmanes, donde puede encontrar más audiencia aunque en competición con otros grupos semejantes que también buscan instaurar el califato. En estos países suelen actuar de forma clandestina ya que en la inmensa mayoría están prohibidos (como en todo Oriente Medio, Asia central y Asia Meridional).

De entre estos países, el caso más interesante podría ser Kirguistán, por la abundancia de militantes que se da, sobre todo, en su región sur, poblada mayoritariamente por uzbecos. Pero, sobre todo, es importante el caso de Pakistán, donde parece que están haciendo mella en la sociedad y que han logrado captar a algunos miembros del Ejército ${ }^{33}$ : "British-Pakistani members of Hizb al Tahrir have been directed to attempt recruiting officers inside Pakistani military" 34 .

Además, el movimiento islamista hace continuos llamamientos a las Fuerzas Armadas para que acaben con ese régimen e instauren un califato:

\footnotetext{
${ }^{28}$ Ver "From Dawa to Jihad: The Various threats from Radical Islam to the Democratic Legal Order", Algemene Inlichtingen en Veiligheidsdienst, 30 de marzo de 2005, en https://www.aivd.nl.

${ }_{29}$ Ver "Annual Report 2010", Algemene Inlichtingen en Veiligheidsdienst, 12 de septiembre de 2011, en https://www.aivd.nl, p. 16.

30 Ver "Dinamarca: La "policía religiosa" de Hizb-ut-Tahrir evita que los musulmanes participen en las elecciones", Minuto Digital, 9 de septiembre de 2011, en http://www.minutodigital.com.

${ }^{31}$ Ver el informe Jordán, Javier y Tarrés, Sol: "Hizb ut-Tahrir ( HuT) en España”, Athena Intelligence Journal, vol. $2, \mathrm{n}^{\mathrm{o}} 2$ (2007), en pp. 13-27.

${ }^{32}$ La última fue organizada en el hotel de la cadena Hilton (como suele ser habitual en Hizb ut Tharir America) "Double Tree - Oak Brook" de Chicago (Illinois), el 26 de junio de 2011, de 14:00 a 17:00, y llevaba por título: "Revolution in the Muslim World: From Tyranny to Triumph".

${ }^{33}$ Ver "Brigadier Ali Khan: Pakistan's dissenting army officer", BBC News South Asia, 23 de junio de 2011, en http://www.bbc.co.uk. Ver también Khan, Nayyer: "An Islamist coup in the offing!", The News Tribe, 31 de enero de 2011, en http://www.thenewstribe.com.

${ }^{34}$ Ver “Brig. Ali Khan Case: British Intelligence Infiltrating Pakistan Army?”, Pakistan Kakhuda Hafiz, 23 de junio de 2011, en http://www.pakistankakhudahafiz.com.
} 
Hizb ut Tahrir calls you to join with it in the movement to establish the Khilafah. It is not difficult to join this work, all you have to do is to reply to the member of Hizb ut Tahrir who carried the call to you or contact our media office. And it calls the Muslim armies to give Hizb ut Tahrir the Nussrah (Material Support) so that Khilafah is established. So, rush to fulfill the great obligation of working to establish the Khilafah, before it is established and you are deprived of the reward for doing so! The decision lies with you, so when will you respond? ${ }^{35}$

Y poco más adelante añade:

In this manifesto, Hizb ut Tahrir Wilayah Pakistan outlines a vision of what the Khilafah will bring for Pakistan and the entire Muslim World. Hizb ut Tahrir calls the Muslims to join with it in the movement to establish the Khilafah. And it calls the Muslim armies to give Hizb ut Tahrir the Nussrah (Material Support) so that the Khilafah is established. ${ }^{36}$

Y de manera más incisiva recientemente:

The solution is clear and that is to work with us and through us to remove these corrupt and secular rulers, to uproot this colonialist kufr system, and implement the laws of Allah through the establishment of the Khilafah. It is only through the Khilafah that you will unify the Ummah, establish the rights of the people, the fair distribution of wealth, return the armed forces to its essential role in the protection of the blood and honour of the Muslims and ensure the removal of the foreign hegemony from the region. This is a duty upon you and if you fulfil it the pleasure of Allah awaits you. But if you delay and turn away, all of the Ummah will be witness upon you on the Day of Judgement, for you strayed away from the solution to the problems of this Ummah. ${ }^{37}$

Parece que en Pakistán, un Estado fallido que se sostiene gracias al interés de EE.UU. por no dejar que caiga en manos de islamistas que escapen de su control, están empujando para derribar lo poco que queda de estructura centralizada de poder del Estado y transformarlo en la primera pieza del califato ${ }^{38}$.

En el Líbano, cuyo sistema político y de partidos es extremadamente complejo debido al equilibrio que debe guardar entre las distintas minorías étnicas y religiosas, se inserta en el sector suní, aunque con escaso éxito. No obstante, es el único país de Oriente Medio en el que opera abiertamente y donde el movimiento está legalizado, ya que la Ley de Asociaciones (que no "de partidos políticos") lo permite. No obstante, a pesar de ser legal y poder operar abiertamente, no se presenta a las elecciones por considerarlas un sistema de infieles. Quizás sería un buen momento para poder saber a ciencia cierta con cuántos apoyos cuenta entre la población suní ${ }^{39}$.

En Indonesia y Bangladesh cuenta con numerosos seguidores, de manera que incluso se les ha acusado ${ }^{40}$ de intentar un golpe de Estado en este último país ${ }^{41}$, golpe frustrado con la

\footnotetext{
${ }^{35}$ Ver "Manifesto of Hizb ut-Tahrir for Pakistan", 24 de febrero de 2009, en http://www.khilafah.com, p. 36.

${ }^{36}$ Ibid., p. 38.

${ }^{37}$ Ver "To the Generals, Air Marshalls and Navy Admirals and officers of the Pakistan armed forces", Hizb ut Tahrir Wilayah Pakistan, 21 de octubre de 2011, en http://www.hizb-pakistan.com.

38 "O sincere officers within Pakistan's armed forces! The shebaab of Hizb ut-Tahrir are fulfilling their pledge before Allah. It is time now for you to fulfill yours. It is time now, O brothers, to grant Hizb ut-Tahrir the Nussrah for the re-establishment of the Khilafah, healing the hearts of the believers and granting them victory over the oppressors". Ver "By abducting the shebaab of Hizb ut-Tahrir, Pakistan's traitor rulers only bring the establishment of the Khilafah even closer", Hizb ut Tahrir Pakistan, 12 de Julio de 2011, en http://www.khilafat.org.

${ }^{39}$ Ver Alonso Marcos, Antonio: “Hizb ut Tahrir en el Líbano...”, op. cit., pp. 201-218.

40 "Though rumours had been circulating about a possible coup for weeks, many in government dismissed the idea, saying the army had no appetite for it under the present circumstances". Ver "Bangladesh army 'foils coup' against Sheikh Hasina", BBC News, en 19 de enero de 2012, en http://www.bbc.co.uk. Ver

${ }^{41}$ Ver Ahmed, Houriya: "Hizb ut-Tahrir and its failed coup in Bangladesh", The Scoop, 20 de enero de 2012, en http://www.henryjacksonsociety.org/thescoop.asp?pageid=106\&poid=1423. Ver también Joseph, Josy: "Hizb ut-
} 
detención del comandante Syed Mohammad Ziaul Huq ${ }^{42}$. La organización respondió publicando un comunicado en el que anuncia la inminente llegada del califato ${ }^{43}$.

\subsubsection{Su papel en la primavera árabe}

Hizb ut Tahrir ha tenido un papel muy activo en las revueltas de la llamada "primavera árabe" y ha puesto muchas esperanzas en esta revolución de los regímenes de los países musulmanes. Así lo ha manifestado en numerosas ocasiones, sea a través de conferencias ${ }^{44}$, panfletos, revistas $^{45}$ o vídeos $^{46}$. No obstante, su temor es que este proceso acabe en una imitación del modelo de democracia occidental y no en califato, que es el sistema que ellos apoyan, basado en los principios islámicos:

The changes in the Middle East, coupled with the crisis in Capitalism, are unprecedented. Once upon a time, Washington, London and Paris were trumpeted as role models for the Muslim world. Now this is not the case - and it means as the debates about the future of the Muslim world begin, Islam will take a central role. ${ }^{47}$

Hizb ut Tahrir apoyó las revueltas en Túnez ${ }^{48}$, Egipto, Libia ${ }^{49}$ y Yemen y está intentando promoverlas en Siria ${ }^{50}$ y Pakistán ${ }^{51}$, además de los países de Asia Central:

Muslims in Libya, like those in Egypt, Tunisia, Syria, Bahrain, Yemen, Algeria and Saudi Arabia, have responded to the Hadith of the Prophet Muhammad (salallahu alaihi wasallam) who said "The

Tahrir, group behind failed Bangla coup plot, growing in stature in India", The Times of India, 24 de enero de 2012, en http://articles.timesofindia.indiatimes.com. Ver por último el análisis de Pattanaik, Smruti S.: "Analysing the failed Coup in Bangladesh", Institute for Defence Studies and Anlyses, 23 de enero de 2012, en http://www.idsa.in.

${ }^{42}$ Ver Sufian, Abu: "Maj Zia spewed militant ideas in army", Bangladesh News 24, 19 de enero de 2012, en http://www.bdnews24.com.

${ }^{43}$ Ver "The establishment of the Khilafah is imminent, by the Will of Allah (swt) and Sheikh Hasina's insanity cannot prevent that", Hizb ut Tahrir Bangladesh, 26 de enero de 2012, en http://www.khilafat.org.

${ }^{44}$ Ver "Khilafah Conference: Uprising in the Muslim world... on the Road to Khilafah", Hizb ut Tahrir Australia, en http://www.uprisings2011.com. El programa de la conferencia, celebrada el 3 de Julio de 2011 en Lidcombe, cerca del centro de Sidney, no deja lugar a dudas sobre la opinión de este grupo en torno a las raíces y el curso que deben tomar estas revoluciones: "Talk I:The Muslim World in the 20th Century: Totalitarian Western Oppression"; "Talk II: An Ummah of Ressistance and Revival"; "Talk III: Uprisings 2011: Hope or Mirage?"; "Talk IV: Western Endeavours to frustrate Muslim Revival"; y "Talk V: all Roads Lead to the Khilafah... the Need of the Time", esta última con el subtítulo: "Can the uprisings serve as a basis towards the liberation and unity of the Msulim world? What role can we play in this?".

${ }^{45}$ Ver Khilafah Magazine, $\mathrm{n}^{\circ}$ 18, octubre de 2011, en http://www.hizb.org.uk.

46 Ver "Arab Spring: Islam or Democracy", Hizb ut Tahrir Britain, 26 de enero de 2012, en http://www.hizb.org.uk. Ver también "One year on: Arab Spring, The 4 Burning Questions", Hizb ut Tahrir Britain, 29 de enero de 2012, en http://www.hizb.org.uk. Además, las sesiones de la tarde de la conferencia internacional celebrada en Londres el 9 de Julio de 2011, titulada "Middle East Revolutions. Whose vision do you support?", se proyectó el documental "Tunisia: Voices from the Street”. Ver "International Khilafah conference, London - 9 July 2011”, Hizb ut Tahrir Britain, 11 de junio de 2011, en http://www.hizb.org.uk.

${ }^{47}$ Ver Khilafah Magazine, ${ }^{\circ} 18$, op. cit., p. 3.

${ }^{48}$ Ver el panfleto "Hizb ut-Tahrir: Tunisia needs the Khilafah system, not just a change of ruler", Hizb ut Tahrir Britain, 16 de enero de 2011, en http://www.hizb.org.uk.

${ }^{49}$ Ver "O beloved people of Libya! O descendents of Umar al-Mukhtar!", Hizb ut Tahrir, 25 de febrero de 2011, en http://www.hizb-pakistan.com. Ver también Mustafa, Taji: "Libya: The Muslim armies have moved, but...", Hizb ut Tahrir Britain, 2 de abril de 2011, en http://www.hizb.org.uk. Ver, por último, "Hizb ut-Tahrir's message to people in Libya on the killing of Gadaffi", Hizb ut Tahrir, 22 de octubre de 2011, en http://www.hizb.org.uk.

${ }^{50}$ Ver "O Muslims of Syria! The Khilafah is the only way to protect your blood and your rights", Hizb ut Tahrir, 5 de mayo de 2011, en http://hizb-pakistan.info.

${ }^{51}$ De hecho, el único estudio sobre un país concreto en la última conferencia internacional sobre el califato celebrada en Londres en julio de 2011 fue el caso de Pakistán, donde últimamente se están centrando muchos esfuerzos por parte del partido. Ver también Khan, Nayyer, op. cit. 
best Jihad is to speak the word of truth against the tyrant ruler". What we have witnessed in the past few weeks has been the beginning of the end of these tyrant regimes. ${ }^{52}$

Simplemente observando las portadas de 2011 Khilafah Magazine, una de sus revistas oficiales (junto con $\mathrm{Al}_{\text {-Waie }}{ }^{53}$ ), se puede comprobar el interés con que han seguido los acontecimientos en estos países y cuáles son sus expectativas. El número de marzo de 2011 aparecía en portada una foto nocturna (y festiva) de la plaza Tharir de El Cairo con el título sobreimpreso "Time for real change" 54 . En mayo de 2011, sobre una foto quemándose del coronel Gadafi, aparecía la frase "Libya in the shadow of Gadaffi" ". El número de julio mostraba un fotomontaje con un mapa del norte de África y Medio Oriente con las fotos de los líderes desde Marruecos hasta Irán y la frase "Global call for change" ${ }^{, 56}$. El último número, el de octubre, mostraba una foto de una de las protestas contra el régimen de Assad en la que los manifestantes golpean con un zapato una fotografía tachada del dirigente sirio, con la frase "Assad: Next in line".

El caso tunecino, tras la caída del dictador, es interesante pues Hizb ut Tahrir deseaba participar en las elecciones ya que se había garantizado que iban a transcurrir bajo el Islam (se entiende, bajo las normas del Islam), pero no les dejaron registrarse como tal partido y tuvieron que pedir el voto para los salafistas ya que, como recoge Al-Jazeera:

Tunisian authorities argue that only parties that respect democracy can be legalised. Belhadj said that his party is not opposed to the electoral process and that, it if had been allowed, it would have taken part in the election. "We didn't refuse to participate in the election for ideological reasons, there are elections in Islam," he [Ridha Belhadj, the party's president] said. ${ }^{57}$

La lucha de Hizb ut Tahrir contra Gadafi y su régimen es mucho más larga y se remonta a los años 70, cuando la organización le envió una carta de protesta tras una reunión mantenida con él durante más de cuatro horas ${ }^{58}$. Desde entonces, Hizb ut Tahrir ha padecido numerosas bajas $^{59}$ entre sus militantes por la defensa del modelo del califato frente al de la revolución de Gadafi, la Yamarihiya, instaurada en 1977.

Con la propagación de su mensaje durante décadas en medio de la sociedad turca, libia o egipcia, Hizb ut Tahrir ha contribuido, junto a otros grupos que preconizan la instauración de un califato, a generar un caldo de cultivo, un ambiente en el que los jóvenes han encontrado una serie de razones por las que luchar contra los tiranos que ocupaban aquellos puestos de poder. Sin embargo, para Hizb ut Tahrir, la "primavera árabe" no debe ser un instrumento para instaurar un régimen democrático sino el califato:

Mere democratic freedom and access to economic opportunities would make developing Egypt untenable. Within such a democratic framework, the proponents have already given assurances of honouring past international security and economic agreements. The revolution has been hijacked in a manner that the existing constraints would be largely untouched. Promoting democracy in the region

\footnotetext{
${ }^{52}$ Ver "Statement from Hizb ut-Tahrir Britain on Western intervention in Libya", Hizb ut Tahrir Britain, 19 de marzo de 2011, en http://www.mykhilafah.com.

${ }^{53}$ Ver http://www.al-waie.org.

${ }^{54}$ Ver Khilafah Magazine, no 15, marzo de 2011, en http://www.khilafah.eu.

${ }^{55}$ Khilafah Magazine, $\mathrm{n}^{\mathrm{o}}$ 16, mayo de 2011, en http://www.khilafah.eu.

${ }^{56}$ Khilafah Magazine, $\mathrm{n}^{\circ}$ 17, julio de 2011, en http://www.khilafah.eu.

${ }^{57}$ Ver "Tunisia's vocal Salafist minority”, Al-Jazeera, 11 de octubre de 2011, en http://www.aljazeera.com.

58 Ver "Communiqué from Hizb ut-Tahrir presented to Colonel Gaddafi", 9 de septiembre de 1978, en http://www.khilafah.com.

${ }^{59}$ Ver una parte de la lista en "Libyan activists of Hizb ut-Tahrir martyred by the dog Gadaffi", Islamic Revival, 22 de febrero de 2011, en http://islamicsystem.blogspot.com. Ver la lista completa en al-Inqadh Magazine, vol. 10, no 37 (septiembre de 1991), pp. 80-105.
} 
has been in the interest of the US for the past decade, so that any new developments could still be manipulatable preserving its interests. ${ }^{60}$

Además "So the desire for rights is clear and the desire for Islam is clear. The people may sometimes use the term "democracy", which will be eagerly seized on by western media networks, but there is clearly awareness that Islam provides the rights that people want." 1 Hay que evitar, según Hizb ut Tahrir, caer en la trampa lingüística de Occidente y no manejar sus mismos conceptos: "The Muslim world is dominated by the West, overtly via military and economic means, but also covertly through the manipulation of language. Control a person's language and you limit his thinking and control his ability to articulate what he desires". ${ }^{2}$

\section{Hizb ut Tahrir en Asia Central}

El papel de Hizb ut Tahrir en Asia Central ha sido tratado por muchos autores. Coinciden, en líneas generales, en señalar que su implantación ha sido exitosa a pesar de las restricciones gubernamentales y de que el Islam originario de allí es el suní de la escuela Hanafi, que es muy tolerante y abierto, además de incluir tradiciones provenientes de otros cultos como el zoroástrico, el tengrismo o el chamanismo, en una muestra más del sincretismo de la zona ${ }^{63}$.

\subsection{1-2001}

Durante los primeros diez años de independencia, el movimiento islamista tuvo que competir con otros grupos que también luchaban por hacerse un hueco en esta región ${ }^{64}$. La nueva situación venía determinada por varios factores: en primer lugar, la caída del régimen comunista soviético, que imponía el ateísmo de Estado y prohibía toda forma de manifestación religiosa, aunque algunas rendijas permitieron que sobreviviera en algunos lugares ciertas formas de culto ${ }^{65}$.

En segundo lugar, los gobernantes de los Estados recién independizados buscaron en sus raíces culturales más profundas una identidad común que les diferenciara de los otros Estados de la región, algo que es una cuestión artificial en una zona que no había conocido

\footnotetext{
${ }^{60}$ Ver Ahmed, Abdullah: "Stealing revolutions with phantom solutions", Khilafah Magazine, $\mathrm{n}^{\circ}$ 16, mayo de 2011, en http://www.khilafah.eu, p. 8.

${ }^{61}$ Ver "Are Muslims calling for democracy or elections?", Hizb ut Tarir, 2 de marzo de 2011, en http://www.hizb.org.uk.

${ }^{62}$ Ver "'Our" revolution", Hizb ut Tahrir, en http://www.uprisings2011.com.

${ }^{63}$ Para un estudio especializado de Hizb ut tahrir en Asia Central, ver Baran, Zeyno (ed.): "The Challenge of Hizb-ut-Tahrir: deciphering and combating radical Islamist ideology", The Nixon Centre, Washington, septiembre de 2004, en http://www.nixoncenter.org; Ver también Karagiannis, Emmanuel: "Political Islam in Uzbekistan: Hizb ut-Tahrir al-Islami”, Europe-Asia Studies, vol. 58, n 2, (marzo de 2006); Karagiannis, E.: "Political Islam in Southern Kazakhstan: Hizb ut Tahrir", Central Eurasian Studies Review,vol. 5 no 1( invierno de 2006), en http://www.cess.muohio.edu; Karagiannis, E.: "Political Islam and Social Movement Theory: The Case of Hizb ut-Tahrir in Kyrgyzstan”, Religion, State \& Society, vol. 33, nº 2 (junio de 2005).

64 Ver "Central Asia: Islam and the State", ICG Asia Report, no 59 (10 de Julio de 2003), en http://www.crisisgroup.org. Ver también "Central Asia: Islamist mobilisation and regional security", ICG Central Asia Report, n ${ }^{\circ} 14$ (1 de marzo de 2001), en http://www.crisigroup.org. También, "Central Asia: Unrest and Instability", ICG Asia Briefing, n 38 (25 de mayo de 2005), en http://www.crisigroup.org. Ver también "Is radical Islam inevitable in Central Asia? Priorities for engagement", ICG Asia Report, $\mathrm{n}^{\circ} 72$ (22 de diciembre de 2003), en http://www.crisigroup.org. Ver, sobre todo, "Radical Islam in Central Asia: Responding to Hizb utTahrir", ICG Asia Report, $\mathrm{n}^{\circ} 58$ (30 de junio de 2003), en http://www.crisisgroup.org.

${ }^{65}$ Ver Kalanov, Komil y Alonso Marcos, Antonio: "Sacred Places and "Folk" Islam in Central Asia", UNISCI Discussion Papers, $n^{\circ}$ 17, en http://www.ucm.es, pp. 176-178.
} 
fronteras nacionales hasta ese momento, que más bien se caracterizaba por la facilidad de paso de un lado a otro. Los dirigentes utilizaron entonces el Islam como instrumento de unificación de la nación, como medio para homogeneizar la identidad de sus naciones.

En tercer lugar, surgió un Islam oficial, controlado por los distintos gobiernos, que poco a poco fue desilusionando a la gente al no dar respuestas a las preguntas ordinarias y cotidianas que surgían ante las nuevas situaciones. Por otro lado, el Islam tradicional tampoco era muy vigoroso, pues había sido prácticamente eliminado en aquellos 70 años de ateísmo impuesto y el número de personas con formación suficiente en el Islam para hacerse cargo de las mezquitas y madrazas era muy reducido. La gente empezó a acudir entonces a personas que se habían formado en el extranjero, generalmente en sitios marcadamente extremistas y deficientes.

En cuarto lugar, se fue pasando paulatinamente del ateísmo impuesto al culto controlado por el gobierno, de manera que muchos de estos grupos, fundamentalmente wahhabistas, se mantuvieron en la clandestinidad.

La llegada de Hizb ut Tahrir a Asia Central se data en 1992, poco después del acceso a la independencia. Aunque la información acerca de este movimiento es escasa y a veces poco fiable, podemos encontrar a través de algunos documentos oficiales los nombres de los responsables de Hizb ut Tahrir en la región: ${ }^{66}$

1992-1996: Abdurashid Hozhimatovich Qasymov.

1996-1999: Hafizulla Muzhahedovich Nasyrov.

1999-2000: Nugman Omonovich Saidaminov.

2000-2001: Istam Igamberdievich Khudoiberdiev.

Algunos autores comparten la idea de que la primera célula de Hizb ut Tahrir apareció en Namangan tras la loiquidación de otras organizaciones islamistas locales tales como Adolat (Justicia), Islam Lashkarlari (El Ejército del Islam), y Tawba (arrepentimiento). Además, señalan que sus líderes eran los jordanos 'Isam Abu Mahmud Qiyadati y Abd al-Qadim Zallum $^{67}$. Esto se trata, sin duda, de un error, pues no es posible situar al líder global de Hizb ut Tahrir como líder del partido en Uzbekistán.

Grosso modo se puede afirmar que, si bien está documentado que en los años $80^{68}$ llegó literatura de Hizb ut Tahrir a estas repúblicas soviéticas, los años $90^{69}$ contemplaron la llegada e implantación de la organización. Siguiendo el relato de Vitaly Naumkin, la primera célula de Hizb ut Tahrir en Asia Central fue creada en Uzbekistán por Abdullamid Bekmuradov. ${ }^{70}$ Éste hizo amistad en la madraza de la principal mezquita de Andiján con Hazifulla Nasyrov. Ambos eran del pueblo de Denau (Uzbekistán) y tuvieron como maestro a Abdulawi qari (Mirzaev). Después de acabar sus estudios en 1995, Nasyrov pasó a Bekmuradov el libro El Estatuto del Islam, de Al-Nabhani. Tanto le impresionó la lectura de

\footnotetext{
${ }^{66}$ Ver Naumkin, Vitaly V. (2005): Radical Islam in Central Asia: between Pen and Riffle, Oxford, Rowman \& Littlefield Publishers, p. 143.

${ }^{67}$ Ver Yemelianova Galina M.: "The Rise of Islam in Muslim Eurasia: Internal Determinants and Potential Consequences”, China and Eurasia Forum Quarterly, vol. 5, n 2 (2007), en http://www.silkroadstudies.org, p. 89.

${ }^{68}$ Ver Naumkin, op. cit., p. 140.

${ }^{69}$ Aunque generalmente se admite 1995 como fecha de inicio de la actividad de Hizb ut Tahrir en la región, otros autores defienden que fue entre 1992-93. Ver Ro'i, Yaacov (2001): Islam in the CIS. A threat to stability?, Central Asian and Caucasian Prospects. Londres, The Royal Institute of International Affairs, p. 32.

${ }^{70}$ Ver Naumkin, op. cit., p. 142.
} 
este libro que decidió unirse al partido, jurando en otoño de 1996, ante Nasyrov, lealtad al partido. Bekmuradov logró reunir en torno a sí en poco tiempo en la región uzbeka de Surkhan Darya a otras tres personas, formando junto con Nasyrov un círculo (halqa) de estudio. En tres años Bekmuradov y Nasyrov dirigían 135 mushrifs, 187 miembros y 337 darises.

En cualquier caso, parece que el lugar de introducción y de mayor auge de este movimiento islamista, y de otros también, es el valle de Fergana ${ }^{71}$.

En esos años, utilizaron los medios habituales de los grupos islamistas para difundir sus ideas: infiltración en mezquitas y madrazas, impartición de conferencias y seminarios, distribución de libros y panfletos, empleo de material gráfico y audiovisual (casetes, videocasetes, CD's, DVD's,...) y, por último, Internet. Además, hay que tener en cuenta que la victoria de los talibanes en Afganistán contra la Unión Soviética en 1989 supuso un acicate y sirvió de ejemplo en los inmediatos años posteriores (aún sigue sirviendo) para demostrar la fuerza de las ideas islamistas frente al poder material de los grandes imperios.

A lo largo de la década de los 90, la represión gubernamental se vuelve más dura y, tras los atentados de 1999 en Uzbekistán, el partido se ve obligado a abandonar todo intento de aparición pública y pasa a la completa clandestinidad. A partir de este momento, viendo Karimov que este grupo servía de alguna manera a los intereses del Movimiento Islámico de Uzbekistán, comienza una dura persecución, primero en solitario, luego con la ayuda de los Estados circundantes (excepto Afganistán, claro está) y, después del 11-S de 2001, de EE.UU. Por eso, algunos han sugerido que en esta época Hizb ut Tahrir Uzbekistán se convence de que debe abandonar la metodología no violenta y aliarse con grupos terroristas para garantizar su supervivencia ${ }^{72}$.

\subsection{1-2011}

A partir de 2001, el apoyo de Occidente a Karimov en su lucha contra el terrorismo es total. Sin embargo, esto no le hace inmune a los atentados terroristas y sufre una serie de ataques en $2004^{73}$. En ese momento se recrudece aún más la represión gubernamental lo que, paradójicamente, se vuelve en contra del propio Karimov ya que, por exceso de celo, encarcela a personas que en realidad no tenían vínculos siquiera con grupos islamistas ${ }^{74}$. Sin embargo, durante su estancia en prisión y tras salir de la cárcel, esas personas pasaron a ser, en su inmensa mayoría, miembros del partido ${ }^{75}$.

Los sucesos de Andiján en mayo de 2005 supusieron otro punto de inflexión. En aquel contexto, la represión policial y de otros cuerpos de seguridad se hizo tan dura que los EE.UU. y la UE decidieron presentar quejas formales e imponer sanciones. Esto trajo como resultado no un arrepentimiento de Uzbekistán sino una búsqueda de nuevos aliados para su

\footnotetext{
${ }^{71}$ Ver Alonso Marcos, Antonio: "Hizb ut-Tahrir (HT) en el Valle de Fergana", UNISCI Discussion Papers, nº 9 (octubre de 2005), en http://www.ucm.es.

72 "It has been suggested by a Western source that the crackdown and mass arrests of 1998 led Hizb al-Tahrir leaders to move from advocating solely peaceful means for achieving their goals to contending that the only effective way to oppose the Karimov regime was trough violence”. Ver Ro'i, op. cit., p. 32. Ver también "Central Asia: Islamist mobilisation and regional security", ICG Central Asia Report, n 14 (1 de marzo de 2001), en http://www.crisigroup.org.

${ }^{73}$ En junio de ese año, la Organización de Cooperación de Shanghái (OCS) decidió establecer en Taskent el centro Antiterrorista Regional (Regional Antiterrorism Structure, RATS).

74 "Amnesty International considers that the charges against Urunboy Usmonov have been fabricated purely as punishment for his journalistic work and for peacefully exercising his right to freedom of expression". Ver "Tayikistán debe liberar a un periodista de la BBC: un caso envuelto en denuncias de tortura", Amnesty International, 7 de julio de 2011, en http://www.amnesty.org.

${ }^{75}$ Ver Naumkin, op. cit., p. 150.
} 
lucha contra el terror, pero siguiendo sus propios métodos. Así, si desde 2001 venía colaborando con la OCS, en 2004 profundiza dicha cooperación y en 2005 recibe en ese foro el respaldo necesario para pedirle a EE.UU. que abandonara su territorio y saliera de Asia Central. A partir de ese momento Hizb ut Tahrir comenzó a ganar cada vez más adeptos y a hacerse fuerte en Uzbekistán y, sobre todo, en Kirguistán y Tayikistán.

Fue precisamente en estos momentos cuando Kazajstán incluyó a Hizb ut Tahrir en su lista de grupos terroristas ${ }^{76}$.

En esta dura etapa para el partido, el jefe de la "provincia" de Uzbekistán es Abdurahim Abdurafukovich Tukhtasinov, según el relato de los propios miembros de la organización en un juicio:

[Mahmud] Karimov said during his trial that, soon after his release, he was approached by one Abdurahim Tukhtasinov, a man responsible for Hizb ut-Tahrir activities in Uzbekistan, who had been on the wanted list on suspicion of committing a number of serious crimes. "After I was released, Abdurahim Tukhtasinov approached me and said that he would send me $\$ 500$ monthly from the money coming from abroad. I was instructed to propagate Hizb ut-Tahrir ideas throughout the Ferghana Valley and recruit new supporters. This was an instruction from one of the organization's leaders, Abu Rashta," Karimov said at the court. ${ }^{77}$

El propio Tukhtasinov ha reconocido la dureza de estos años en una carta abierta:

I sent you part of the treatment that has been inflicted on us by the ruling authority in Uzbekistan; the prosecution, imprisonment and torture which amounts to death in many cases. Such treatment is not limited to us only in Hizb ut-Tahrir but includes all of the workers for the Islamic Call. The number of prisoners from Hizb ut-Tahrir alone in the prisons of Uzbekistan is approximately eight thousand, even though that party does not exert any acts of violence. The oppression of this government was aggravated more this summer than throughout the year. At the beginning of this summer, all the new masools (leaders) who were appointed after the arrest of their predecessors have been arrested. A large number of our active shabab were also arrested. This happened in Tashkent, the capital, in the state of the Ferghana Valley, the state of Andijan and in other states of Uzbekistan. ${ }^{78}$

Tanto el Movimiento islámico de Uzbekistán como Hizb ut Tahrir se nutren principalmente de jóvenes desempleados e incluso con poca formación aunque abarcan a grupos más amplios de descontentos con la situación económica-social de la región. Es difícil precisar el número de seguidores reales de la organización, especialmente si se desea hacer la diferenciación entre simpatizantes y militantes:

Although it is impossible, given the dearth of verified data, to establish the actual membership of HT, Akramiyya, the IMU, and other small Islamist organisations, it seems plausible that those organisations unite between thirty and fifty thousand active members. In addition, the relatives of the activists constitute a much larger group of sympathizers. ${ }^{79}$

\footnotetext{
76 "Hizb ut-Tahrir has already been banned in Uzbekistan, Tajikistan, and Kyrgyzstan, as well as in Russia. Those governments also regard it as an extremist organization". Ver Saidazimova, Gulnoza: "Kazakhstan: Government Moves To Add Hizb Ut-Tahrir To List Of Terror Groups", Radio Free Europe/Radio Liberty, 18 de marzo de 2005, en http://www.rferl.org.

${ }^{77}$ Ver "Uzbekistan sentences Hizb ut-Tahrir leader, accomplices to lengthy prison terms", Interfax, 22 de abril de 2009, en http://www.interfax-religion.com.

${ }^{78}$ Ver "A letter from the Leader of Hizb ut-Tahrir in Uzbekistan", Hizb ut Tahrir, 20 de septiembre de 2010, en http://www.khilafah.com.

${ }^{79}$ Ver Yemelianova Galina M.: "The Rise of Islam in Muslim Eurasia: Internal Determinants and Potential Consequences", China and Eurasia Forum Quarterly, vol. 5, nº 2 (2007), en http://www.silkroadstudies.org, p. 88.
} 
En esta década, han sido detenidos ${ }^{80}$ algunos miembros en Kazajstán ${ }^{81}$, en Kirguistán ${ }^{82}$, Tayikistán $^{83}$, Turkmenistán ${ }^{84} \mathrm{y}$, por supuesto, en Uzbekistán ${ }^{85}$. Precisamente es en las naciones donde la situación social está más deteriorada (Kirguistán y Tayikistán) donde se han practicado más detenciones ${ }^{86}$.

\section{Conclusiones}

Hizb ut Tahrir ha demostrado su capacidad de adaptación a las diversas situaciones históricas manteniendo, eso sí, su ideología y metodología de manera constante, con las excepciones de los intentos de golpe de Estado en los años 60 y $70^{87}$.

En Asia Central ha demostrado tener una presencia fuerte, aunque perdió la oportunidad de las revueltas en Kirguistán de los años 2005 y 2010 y las elecciones de 2011 para hacerse más presente en la vida pública y optar a puestos de representación, ya que, si bien están prohibidos en los cinco países, podría haber aprovechado para solicitar su legalización, al menos en esta nación.

Su presencia ha ido creciendo con el paso del tiempo y, a pesar de los intentos de los gobernantes de reducir su influencia, el hecho es que van siendo cada vez más populares entre

\footnotetext{
80 "The request to outlaw Hizb ut-Tahrir in Kazakhstan comes as 15 people are on trial in the southern Taraz Oblast. The defendants -- accused of plotting last year's attacks in Uzbekistan -- are alleged to be members of Jamoat Mujahedin, or Community of Holy Warriors, an Islamic group with alleged links to Hizb ut-Tahrir". Ver Saidazimova, Gulnoza: "Kazakhstan: Government Moves to...", op. cit.

81 " [...] in Mangistau Region (western Kazakhstan). Five members of the Hizb-ut-Tahrir cell were detained "during a regular clandestine meeting at a flat belonging to one of them. As a result of searches, two computers and a substantial amount of extremist literature were seized." This included 100 journals and brochures, more than 400 leaflets, and 65 CDs. "It has been established that the party cell in Zhanaozen [a town in the region] was set up in 2006 on an order from Hizb-ut-Tahrir leaders in Kazakhstan, who are now on trial in Karaganda" . Ver McDermott, Roger: "Kazakhstan Cracking down on Hizb-Ut-Tahrir", Eurasia Daily Monitor, vol. 4, nº 162 (4 de septiembre de 2007), en http://www.jamestown.org.

${ }^{82}$ Ver "Kyrgyz security services detain one of the most active Hizb ut-Tahrir leaders", 24.kg News Agency, 11 de enero de 2011, en http://eng.24.kg.

83 ""Five active party members were detained in an apartment in Dushanbe which was a meeting place for Hizbut-Tahrir members. Some 100 books, over 200 leaflets and 116 video CDs with extremist contents as well as a computer and flash cards with original information were seized during a search in the apartment [...] In 2010 the courts of Tajikistan sentenced 158 people to varying prison terms on charges of religious extremism". Ver "Five religious extremists detained in Tajikistan", Kyiv Post, 4 de enero de 2012, en http://www.kyivpost.com.

84 "There have also been occasional reports of Hizb ut-Tahrir work in Turkmenistan's prisons". Ver "Radical Islam in Central Asia: Responding to Hizb ut-Tahrir", op. cit., en http://www.crisisgroup.org., p. 14. Ver también Akbarzaden, S.: "Political Islam in Kyrgyzstan and Turkmenistan”, Central Asian Survey, vol. 20, n 4 (2004), p. 455 .

${ }^{85}$ Ver Agakhanov, Farid: "Members of the banned Hizb ut-Tahrir organisation arrested in Tashkent", Central Asia Online, 18 de agosto de 2008, en http://centralasiaonline.com. Ver también el informe elaborado por Hizb ut Tahrir sobre el caso de muerte por tortura de Farhad Usmanov; ver "Persecution of Hizb ut Tahrir Uzbekistan", Hizb ut Tahrir, junio de 2002, en http://www.war-against-terrorism.info.

86 "Tajikistan alone detained some 200 members of extremist and terrorist organizations, and secured convictions against about 170 of them in 2011. Among those detained, 86 people were members of the IMU, 17 were members of the radical Islamist group Hizb-ut-Tahrir (HT) and four others were involved in the activities of the Islamic group Tablighi Jamaat, which was declared extremist by Dushanbe in 2006". Ver "Terrorism: ISI's secret empire in Central Asia", 21 de enero de 2012, en http://windowonheartland.blogspot.com.

87 "In 1968 Hizb ut-Tahrir made an attempt to overthrow the governments in Jordan and Syria, which failed. The organisation also intended to carry out a putsch in Egypt in 1974, and the man who killed Anwar Sadat in 1981 was a member of the group. Attempts at a coup d'etat were also made in Iraq and Tunisia. In 1994 ten members of Hizb ut-Tahrir were accused of planning to kill Jordan's King Hussein". Ver Agakhanov, op. cit.
} 
las masas de descontentos que, especialmente en tiempos de crisis, se han incrementado en esta región.

La situación de Afganistán y Pakistán y la presencia estadounidense en la zona han afectado negativamente a la estabilidad de estos países pues el flujo de tráficos ilícitos, que a su vez nutre a los grupos islamistas, es prácticamente imparable en una zona con unas fronteras tan porosas.

Sólo la aislada Turkmenistán y la próspera Kazajstán parecen librarse del azote de este grupo, ya que su presencia en dichos países es mínima (apenas unos cientos). 